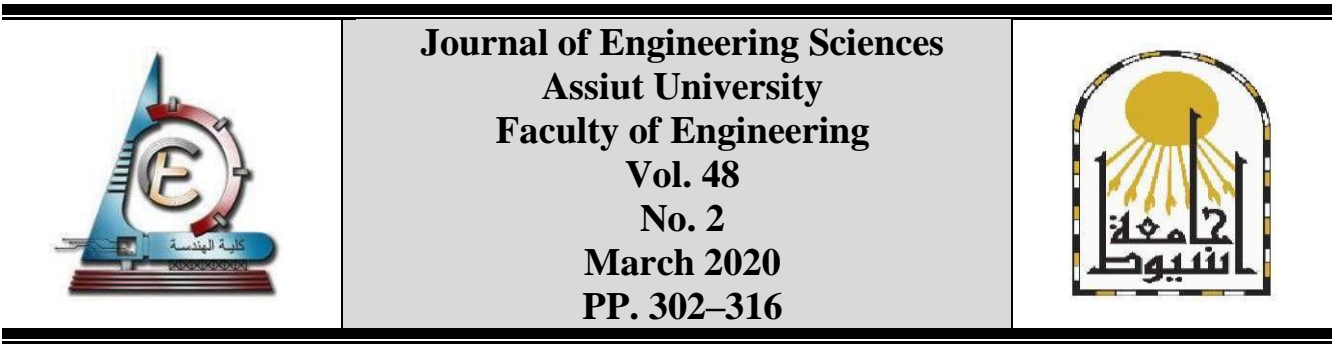

تأثير المو اد متغيرة الطور على كفاءة استهلاك الطاقة بمباني المناطق الحارة الصحراوية

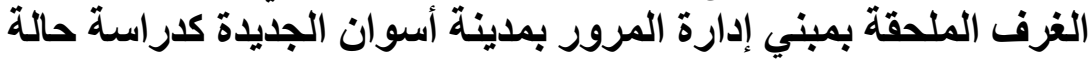

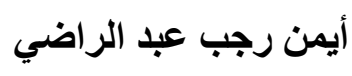

قسم الهندسة المعمارية، كلية الهندسة، جامعة أسوان

Email address:ayman.ragab@aswu.edu.eg

Received 28 August 2019; Accepted 10 September 2020

الملخص

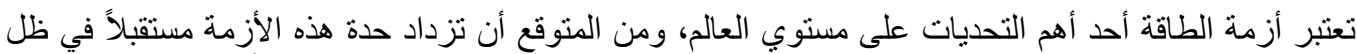

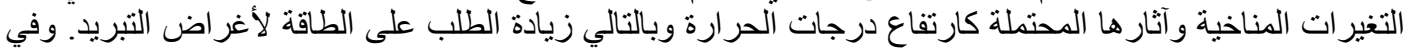

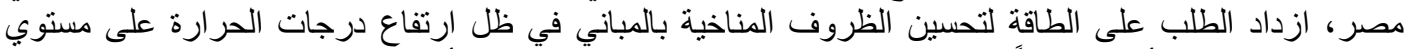

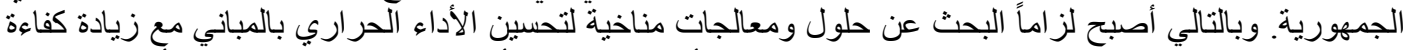

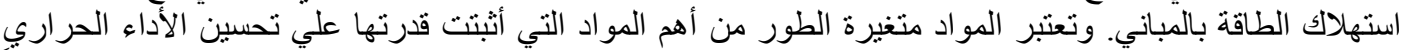

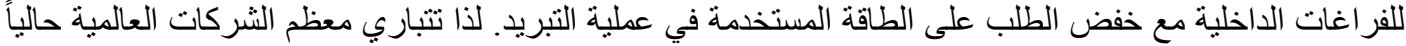

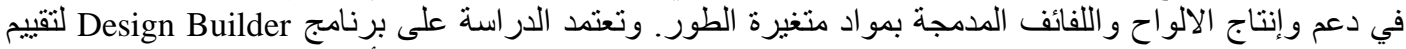

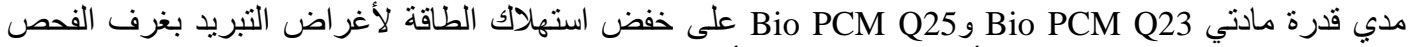

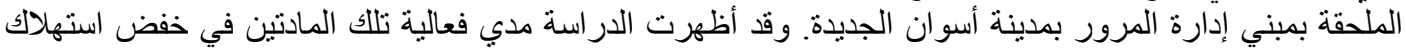

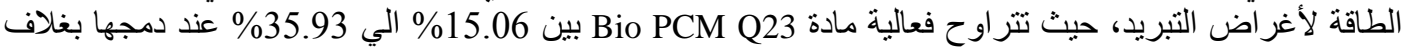

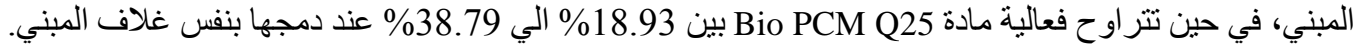

الكلمات المفتاحية: المو اد متغيرة الطور ـ كفاءة استهلاك الطاقةـ غلاف المبني- Design Builder.

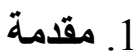

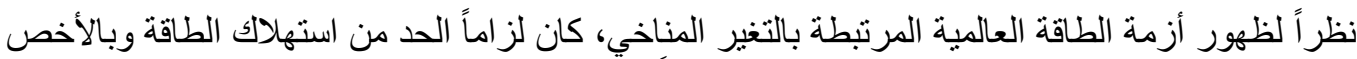

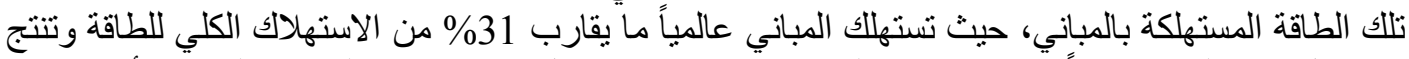

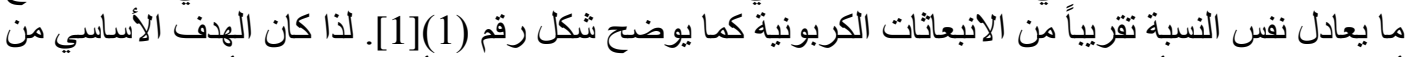

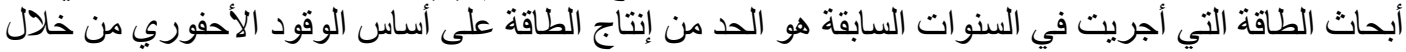

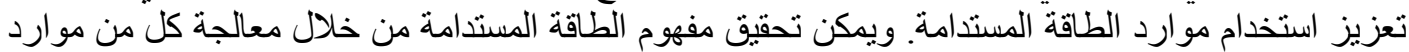

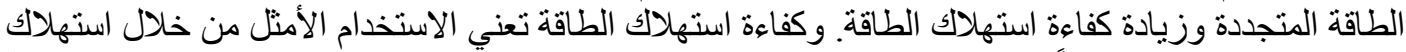

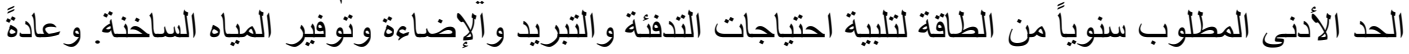

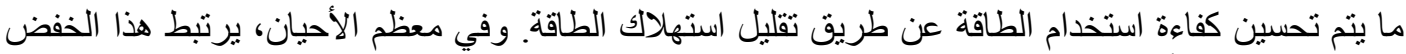

بالحد من استخدام أجهزة التبريد و الاعتماد على تطبيقات التبريد السلبي.

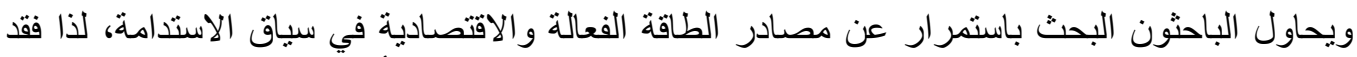

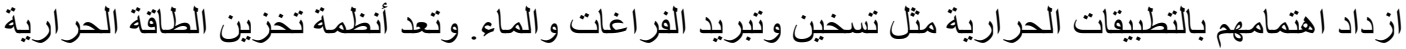
المعتمدة على المواد متغيرة الطور (PCM) Phase Change Materials واحدة من التقنيات الر ائدة لتطوير الطئ 


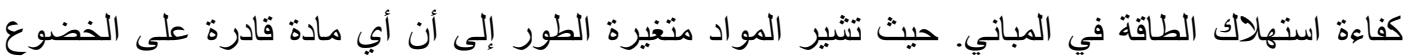

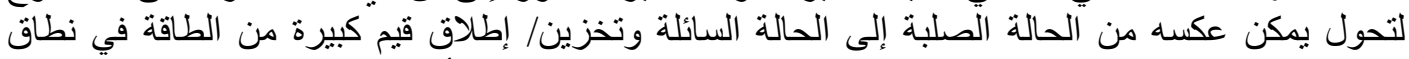

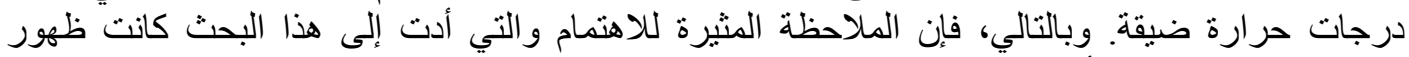

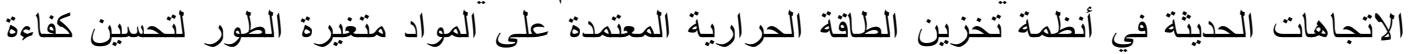

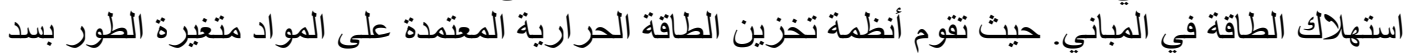

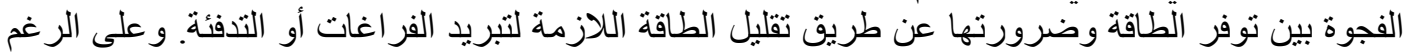

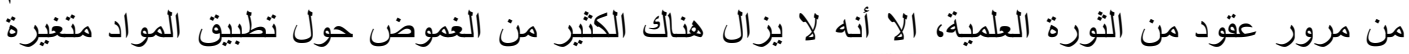

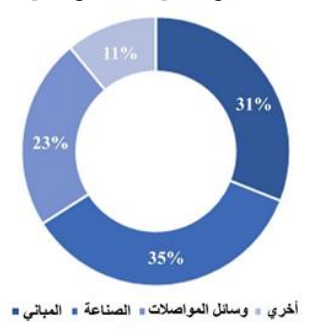

(ب)

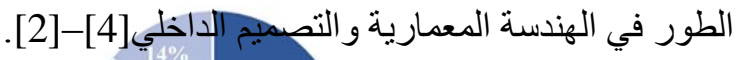

شكل رقم (1): (أ) استهلاك الطاقة العالمي، (ب) الانبعاثات الكربونية العالمية][1]

1.1 إثكالية البحث 1.1

تتبع إثكالية البحث نتيجة عدم قدرة المباني بتصميمها الحالي على تحقيق الاستقرار الحراري الداخلي لها.

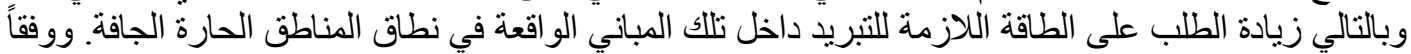
لذلك، يتتافس المهندسون المعماريون على إيجاد تصميم قابل للتطبيق في استر أتيجية التحكم في طاقة المبان المباني.

$$
\text { 2.1 أهمبة البحث وأهد/فه }
$$

يسعي البحث نحو دراسة تأثير استخدام بعض المنتجات التجارية للمواد متغيرة الطور عند دمجها بأغلفة

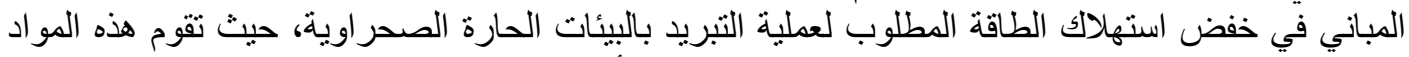

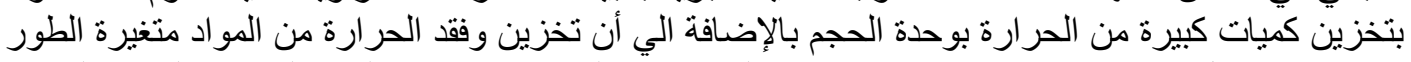

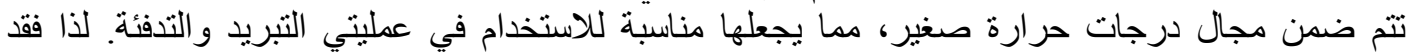

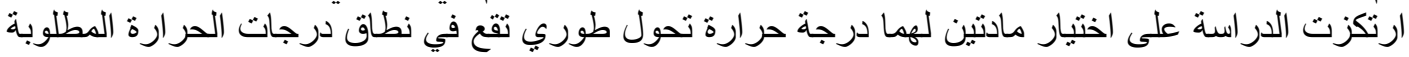

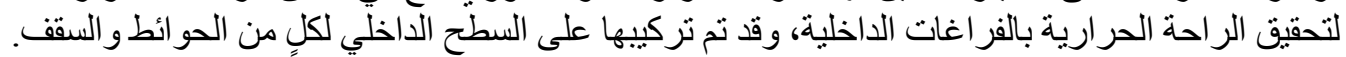

$$
\text { 3.1. منهجية البحث }
$$

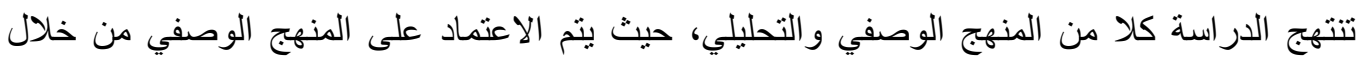

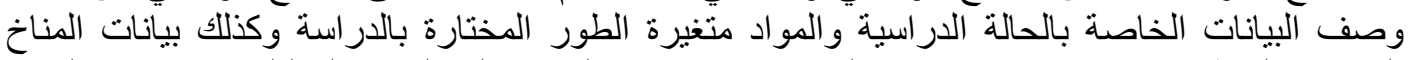

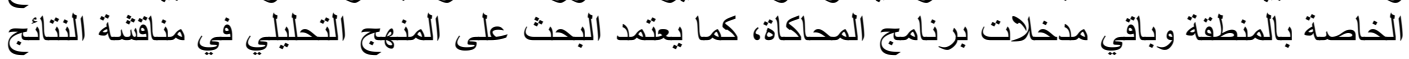

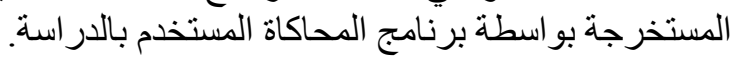

ويستند البحث بشكل أساسي على برنامج المحاكاة [Design Builder[5 في دراسة مدي تأثثر ادماج

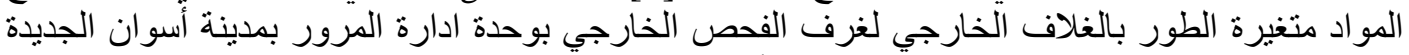

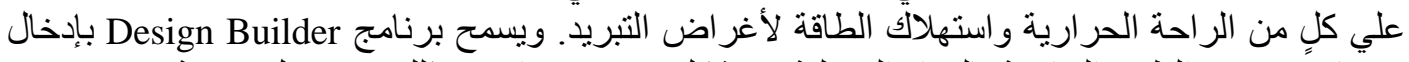

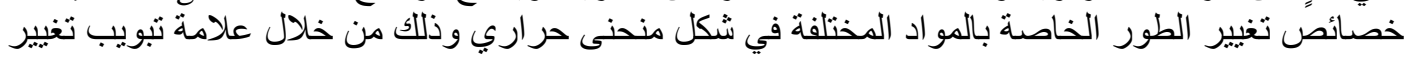

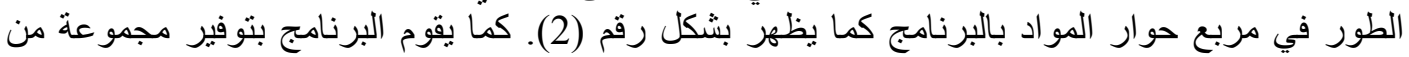

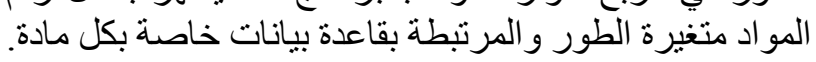




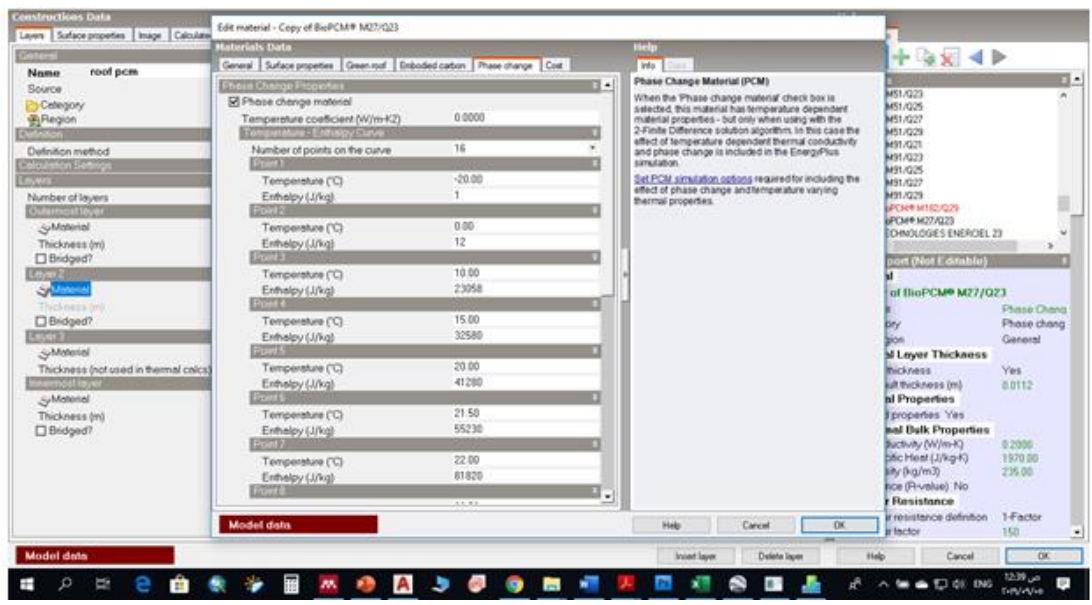

شكل رقم (2): كيفية إدخال خصائص تغيير الطور الخاصة بالمو اد المختلفة ببرنامج المحاكاة.

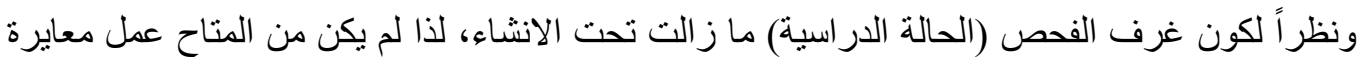

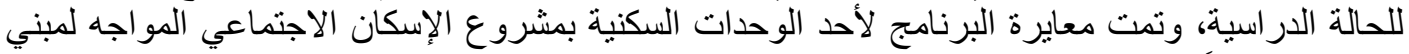

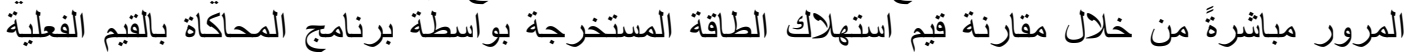

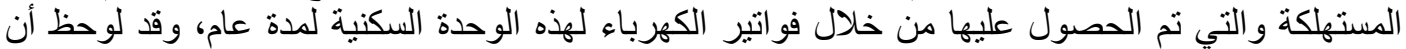

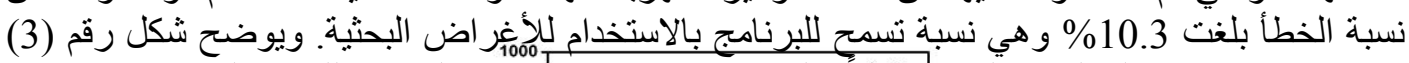

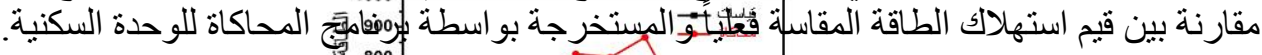

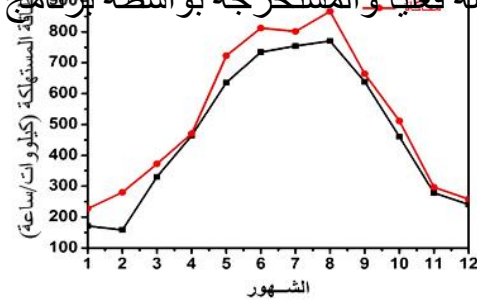

شكل رقم (3): المعايرة غير المباشرة لنموذج الدر اسة و الذي حقق نسبة خطأ 10.3 ٪.

\section{2. ماهية المواد متغيرة الطور}

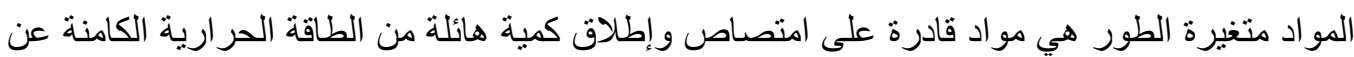

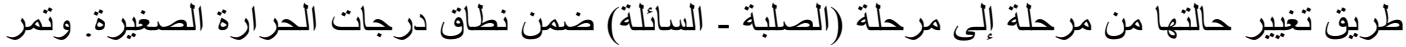

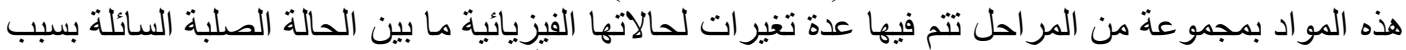

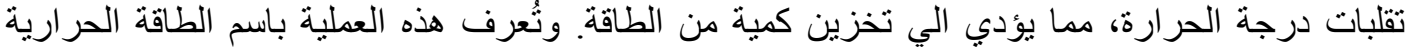

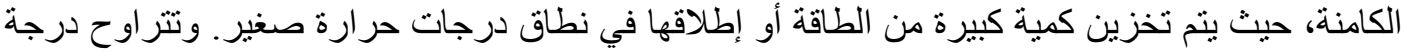

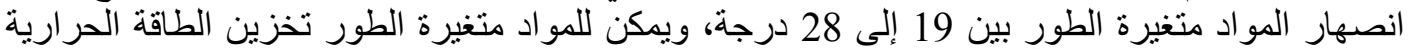

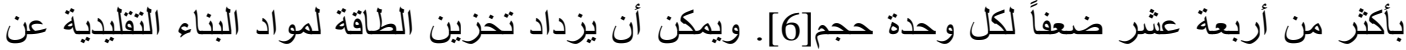

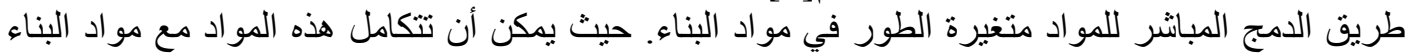

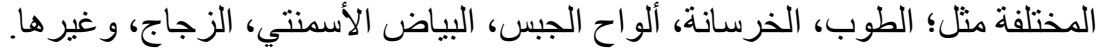




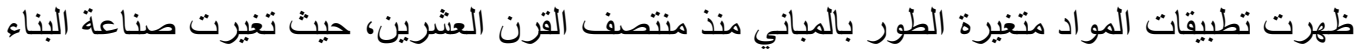

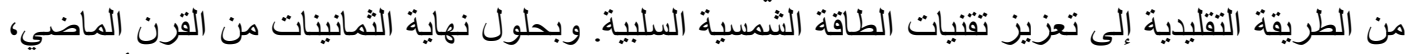

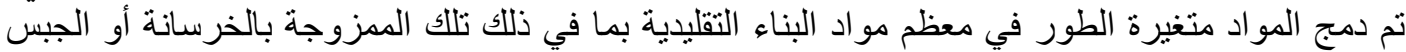

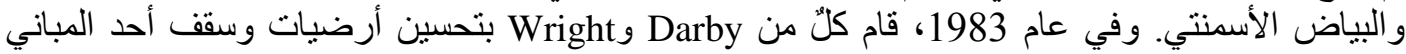

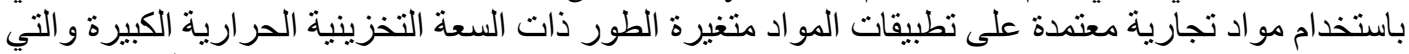

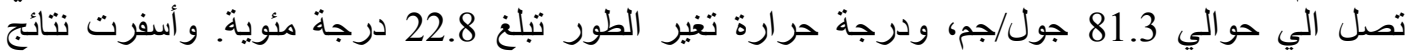

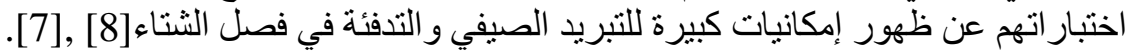

وفي عام 1990، تم تطوير مو اد متغيرة الطور أكثر فعالية من حيث التكلفة كما أنها مقبولة بيئياً بواسطة

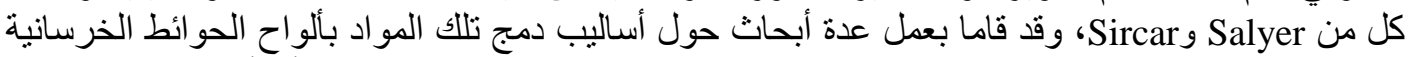

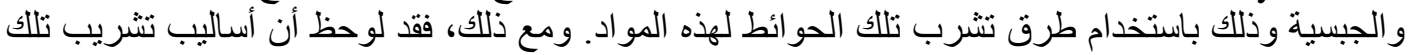

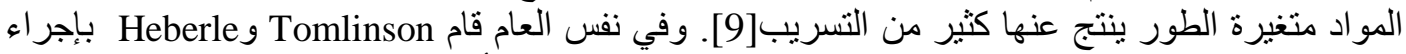

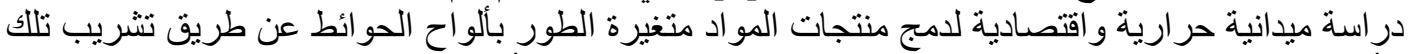

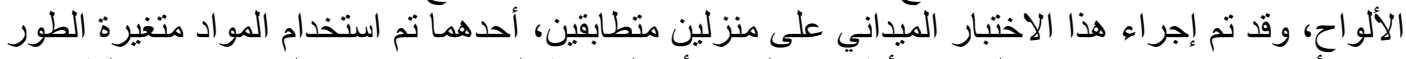

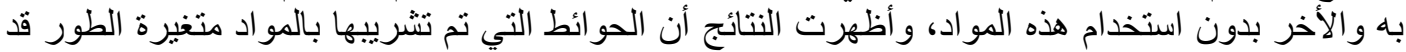

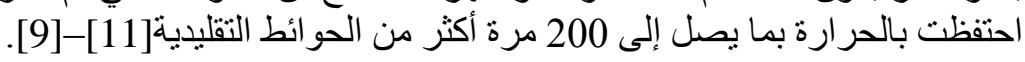

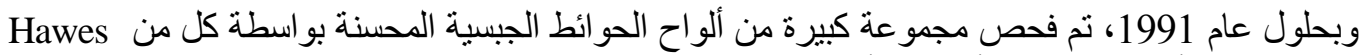

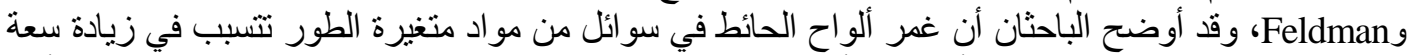

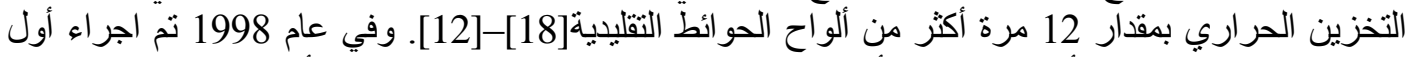

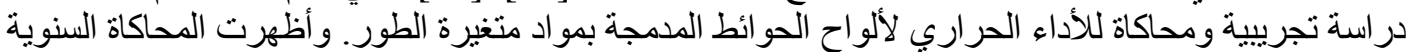

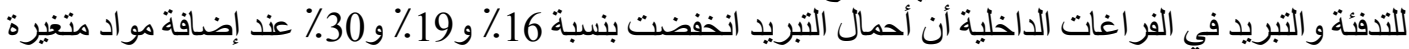

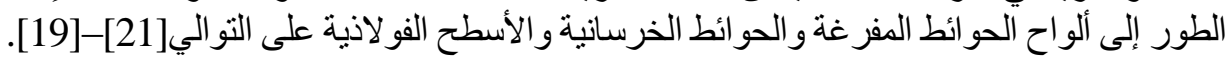

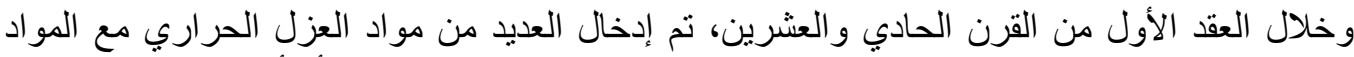

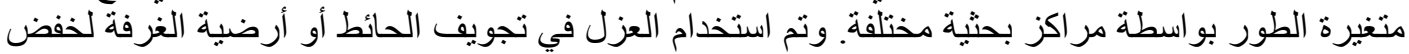

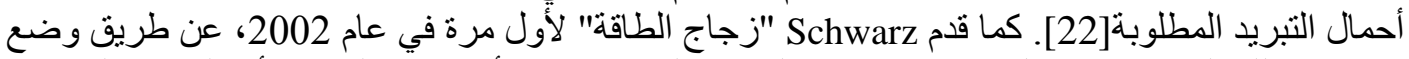

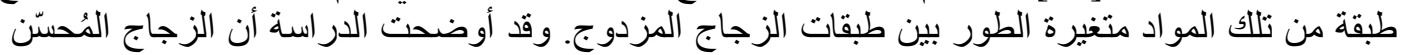

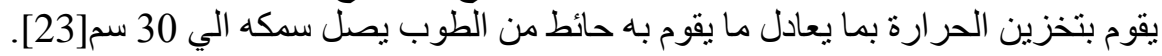

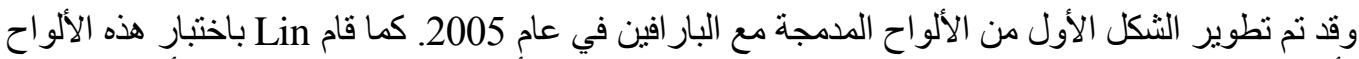

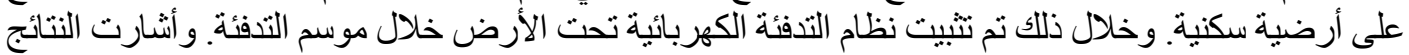

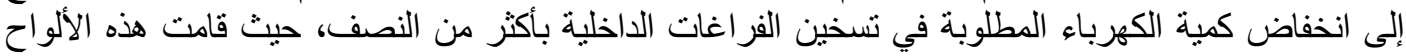

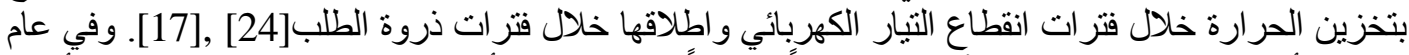

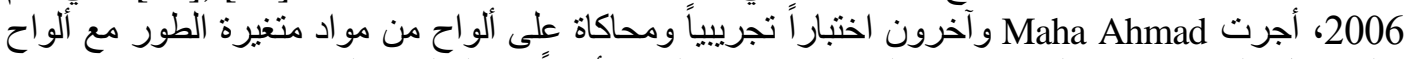

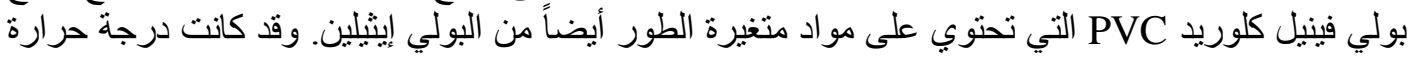

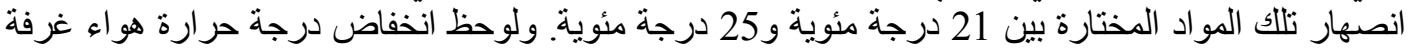

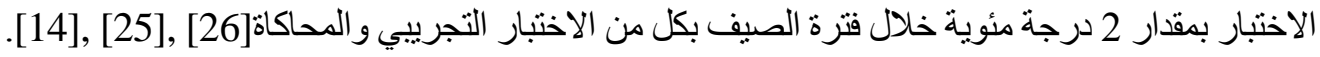

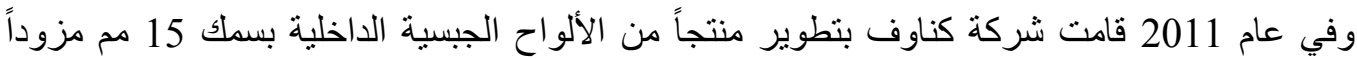

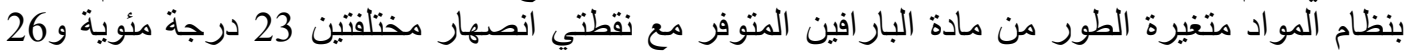

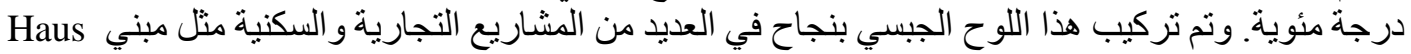

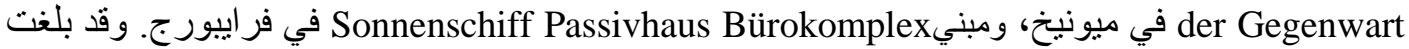

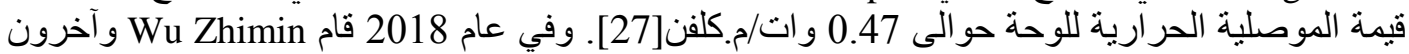

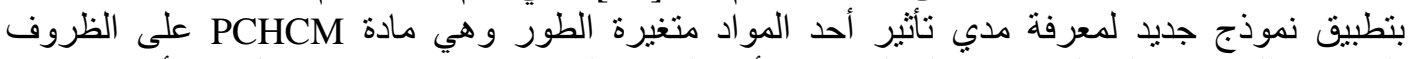
الحر ارية للفر اغات الداخلية واستهلاك الطاقة في أحد المباني المكتبية بمجمو عة مدن تمثل عدة أنماط مناخية 


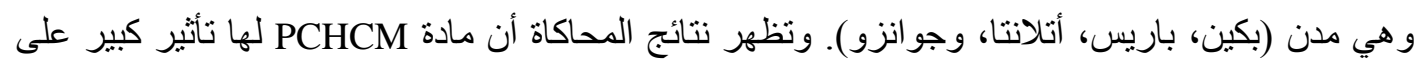

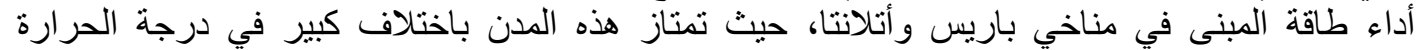

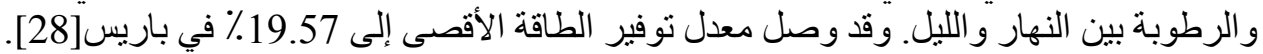

\section{4. تصنيف المواد متغيرة الطور ومعايير اختيارها}

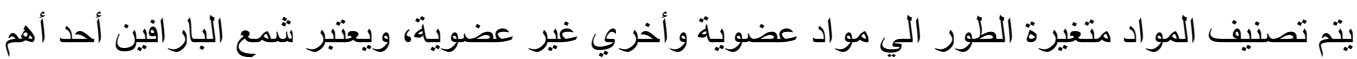

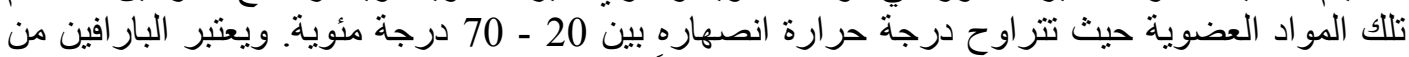

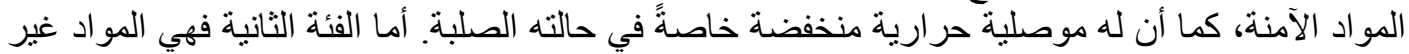

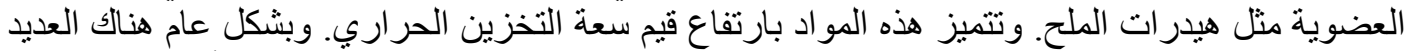

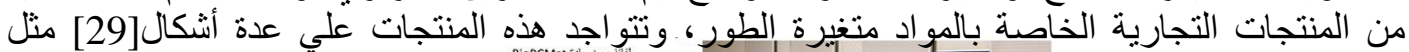

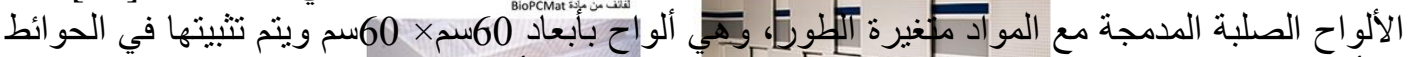

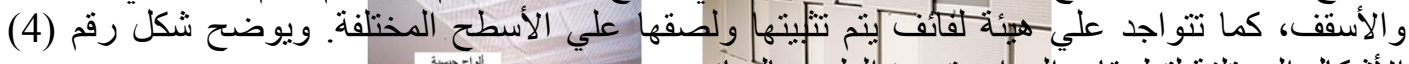

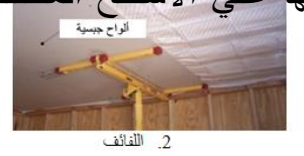

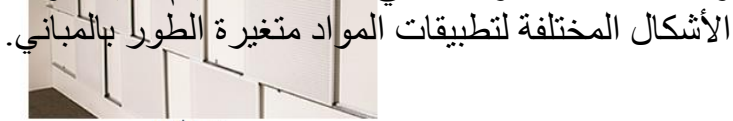

شكل رقم (4): الأشكال المختلفة لتطبيقات المو اد متغيرة الطور بالمباني[30].

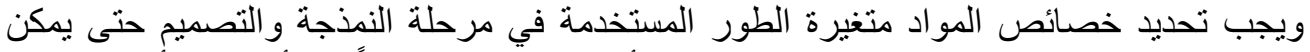

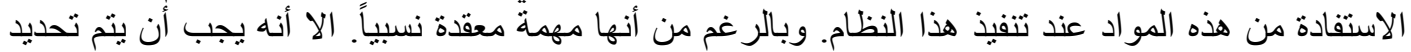

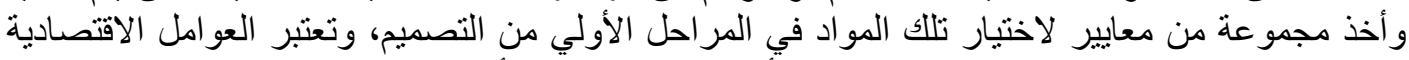
و الخصائص الحر ارية والفيزيائية والكيميائية من أهم المعايير الواجب الأب أخذها في الاعتبار[ [31].

$$
\text { 1.4 الخصائص الحرارية }
$$

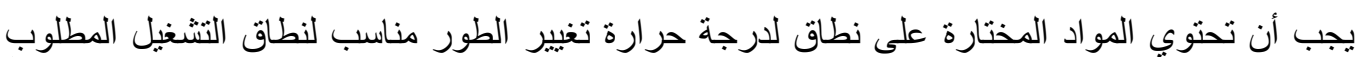

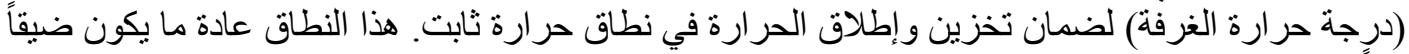

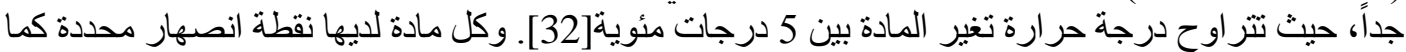

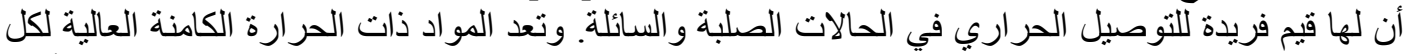

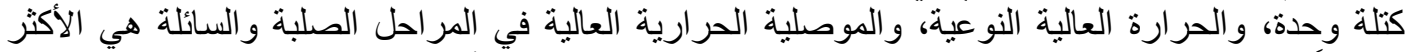

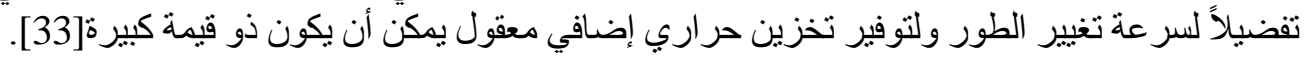

$$
\text { 2.4 الخصائص الفيزيائية }
$$

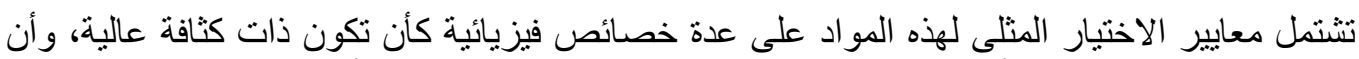

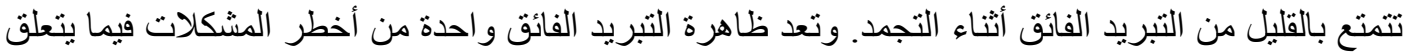

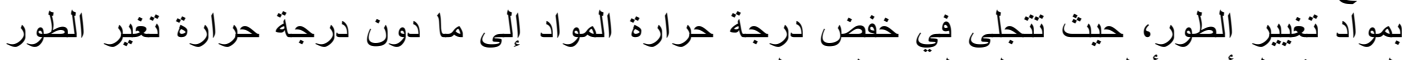
المتوقعة قبل أن تبدأ المادة في التصلب و في فلطلاق الحر ارة [34].

$$
\text { 3.4. الخصائص الكبيبائية }
$$

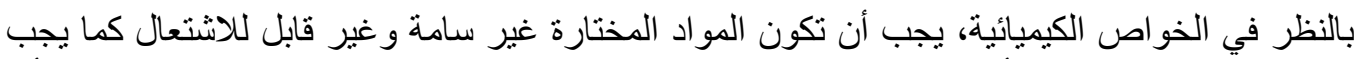

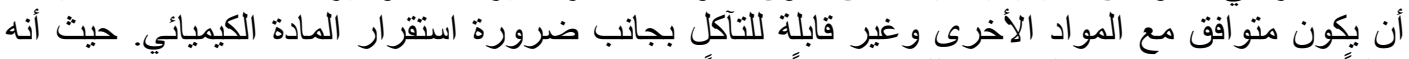
غالباً ما يضمن الاستقر ار الكيميائي للمو اد عمر اً طويلاً [34]. 


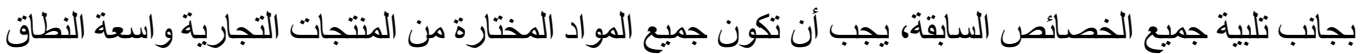

منخفة التكاليف كما يجب أن يكون منوفر على نطاق واسع بجانب وضوح الأداء البيئي العام الجيد لهذه المو اد.

5. تأثير المواد متغيرة الطور على استهلاك الطاقة بالبيئات الحارة الجافة (الحالة الدراسية)

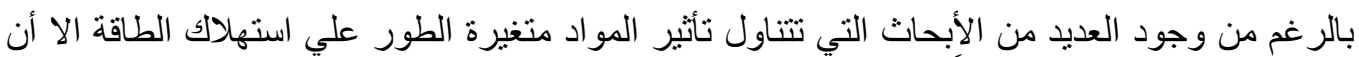

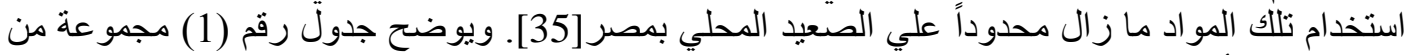

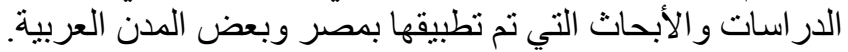

جدول رقم (1): الدر اسات السابقة المطبقة بمصر وبعض المدن العربية.

\begin{tabular}{|c|c|c|c|c|c|}
\hline المرجع & الثنتأبَج & طريتة الثمنجة & 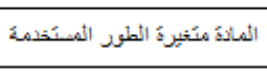 & الثناقي المكاتئ & 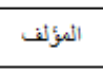 \\
\hline [36] & 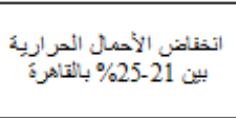 & 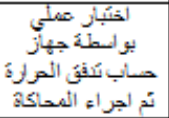 & - Bio-based Pcm & 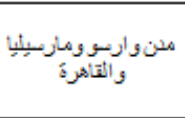 & $\begin{array}{l}\text { Jan } \\
\text { Kosny } \\
\text { وخَرون }\end{array}$ \\
\hline$[22]$ & 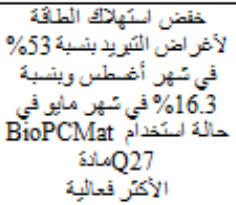 & 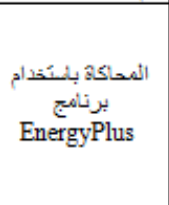 & $\begin{array}{ll}\text { - } & \text { Weber.mur.clima. } \\
\text { 26 plaster mix } \\
\text { - } & \text { KnaufPCM } \\
\text { - } & \text { BioPCMat Q27 }\end{array}$ & مبتي الثاهيرية بمدينة & رباب فكري \\
\hline$[37]$ & 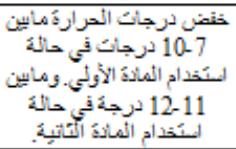 & 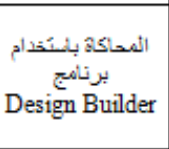 & $\begin{array}{ll}- & \text { M91/Q27 } \\
- & \text { M182/Q27 }\end{array}$ & مبنى نُجازري بمدينة & نيوسف لإحن \\
\hline$[38]$ & 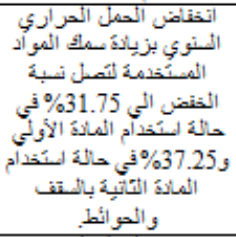 & 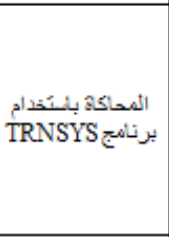 & $\begin{array}{ll}- & \mathrm{C} 16 \\
- & \mathrm{RT} 27\end{array}$ & 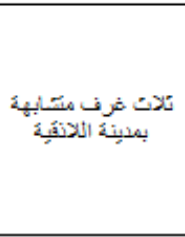 & 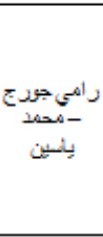 \\
\hline [39] & 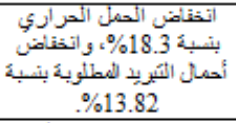 & 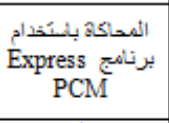 & - PCM23 Micronal & وحلةً سكمنة بمدينة & مها أحمد - مثل \\
\hline$[40]$ & 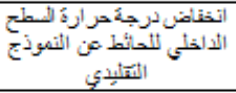 & 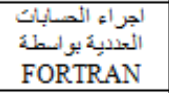 & - - تسمع الثيرافين & بغداد & برذلني \\
\hline [41] & 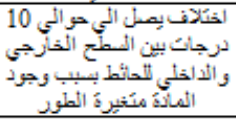 & 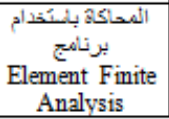 & - $\quad$ ClimSel C21 & 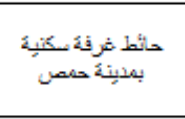 & المحتوضن \\
\hline
\end{tabular}

وبعد استطلاع الأبحاث السابقة الخاصة باستخدام بعض المواد متغيرة الطور بمصر وبعض البلدان

العربية، يقوم البحث باختبار نوعين من المواد متغيرة الطور هما Bio PCM Q23 ومادة وذللك بدمج كليهما على حده بكل من سقف وحو ائط غرف الفحص الخدارجي بمني الفي إدارة المرور بمدينة

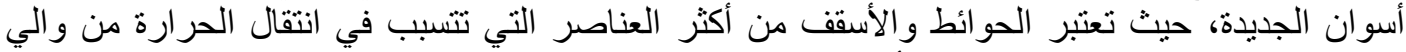

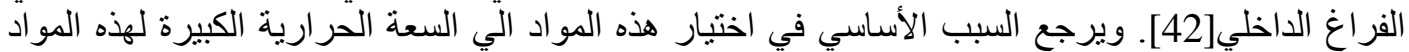

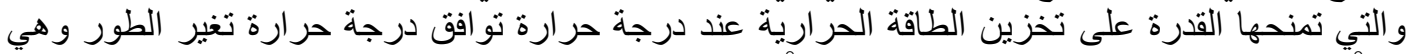

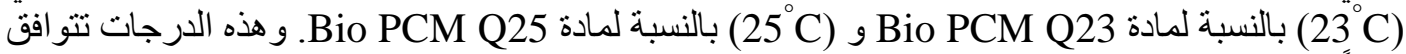

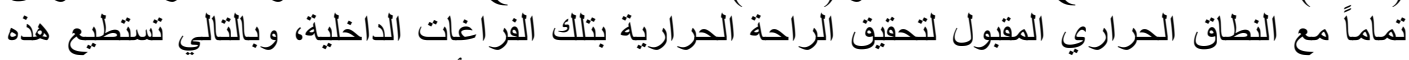

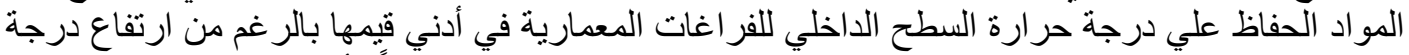
حرارة سطحه الخارجي[46]-[43] , [31]. كما نتوافر هذه المنتجات تجارياً بأسعار منخفضة على على هيئة 


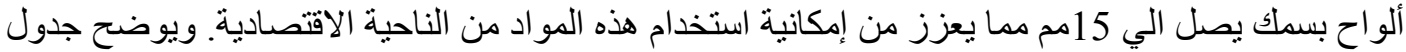
رقم (2) الخصائص الفيزيائية والحرارية المختلفة لتلاك المو اد المستخدمة.

جلول رقم (2): الخصائص الفيزيائية و الحر ارية للمو اد المختبرة[47].

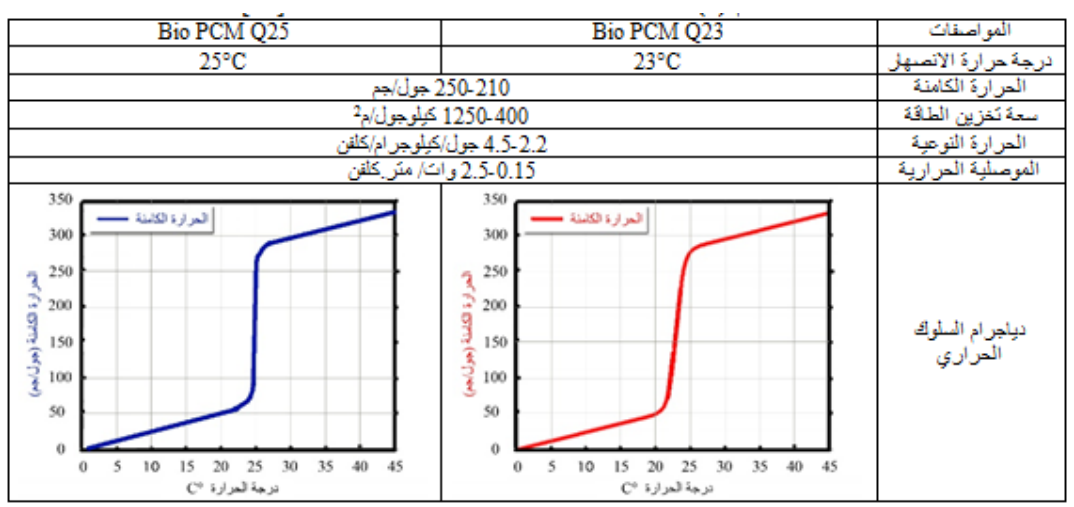

يقوم البحث بعمل نموذج المحاكاة بنفس مو اصفات الحالة الدراسية على أرض الوالو اقع (الحالة الأساسية)، كما

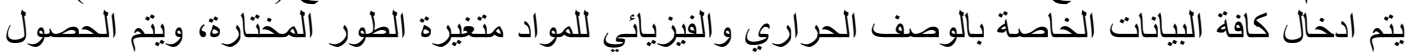

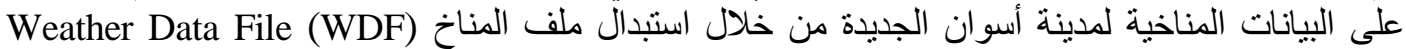

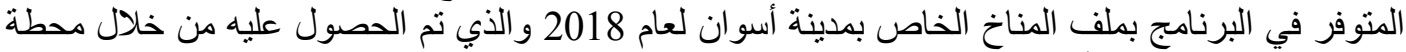

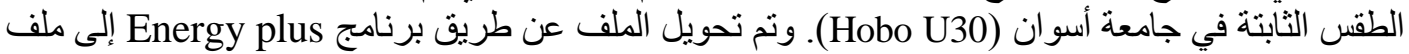
epw ليكون ملائم لتحميل البيانات بالبرنامج. وفيما يلي وصف لكل من منطقة ونموذج الدر اسة.

\section{5. وصف منطقة الدر/سة}

أجريت الدر اسة على إحدى غرف الفحص الملحقة بمبني إدارة المرور بمدينة أسوان الجديدة، وتقع مدينة

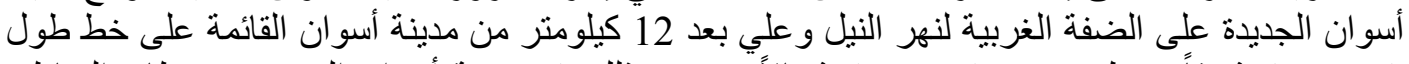

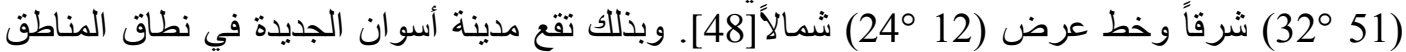

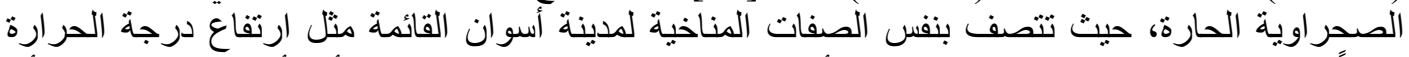

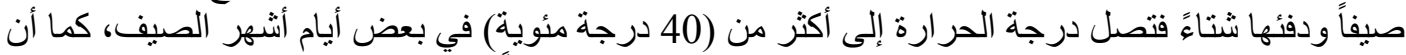

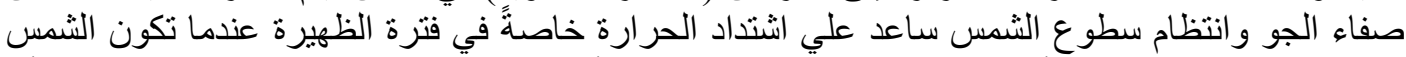

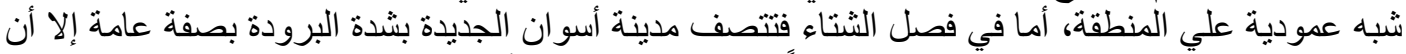

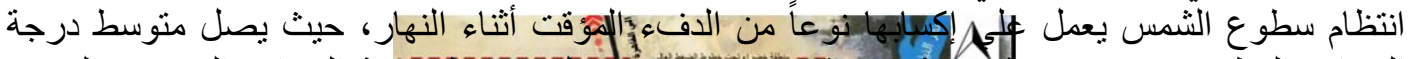

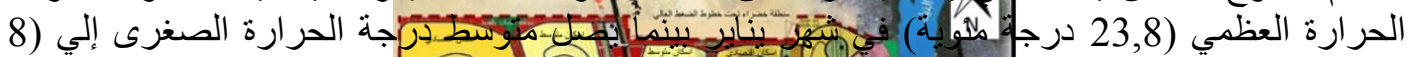

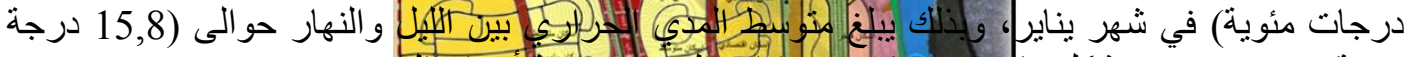

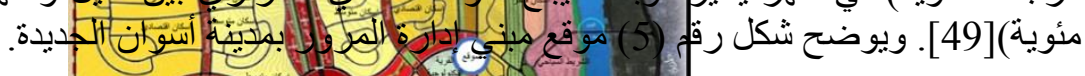

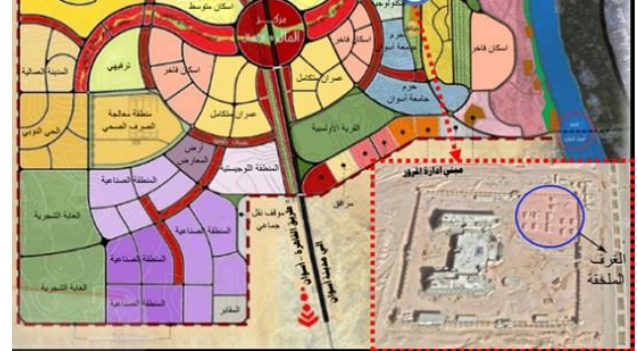


شكل رقم (5): خريطة توضح موقع مبني إدارة المرور بمدينة أسوان الجديدة[48].

$$
\text { 2.5 وصف نموذج الدراسة }
$$

تم تخصيص غرف فحص السيارات الملحقة بمبني إدارة المرور بمدينة أسوان الجديدة للفنبين وعناصر

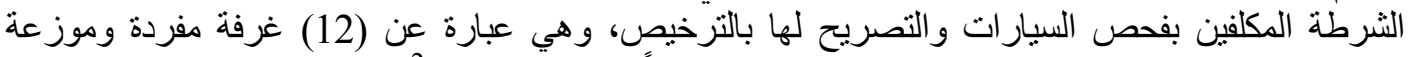

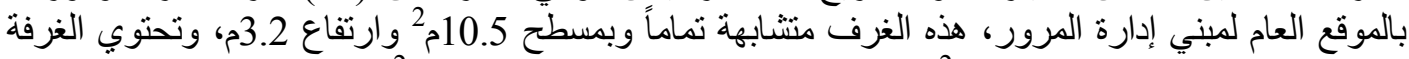

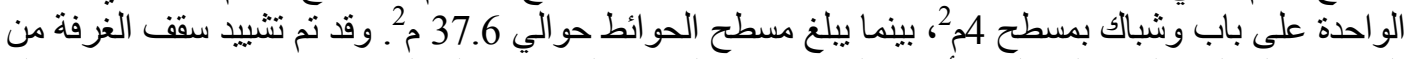

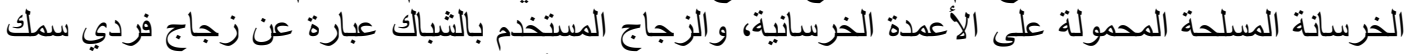

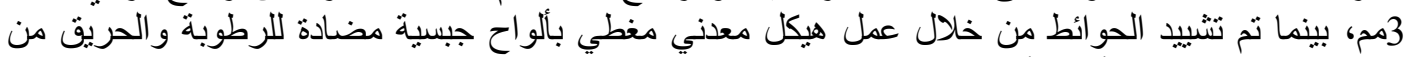

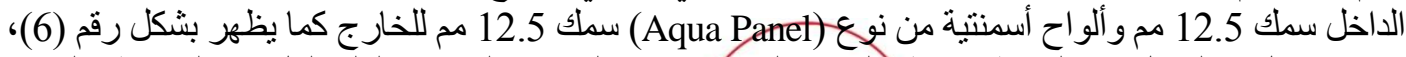

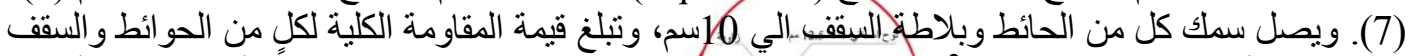

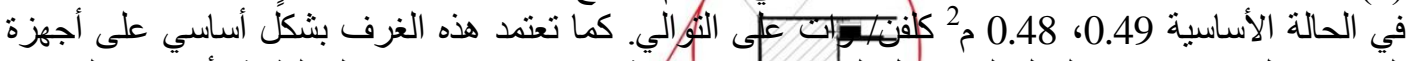

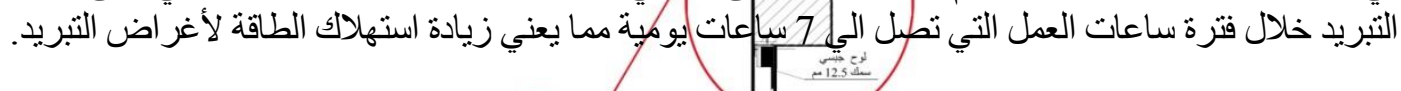
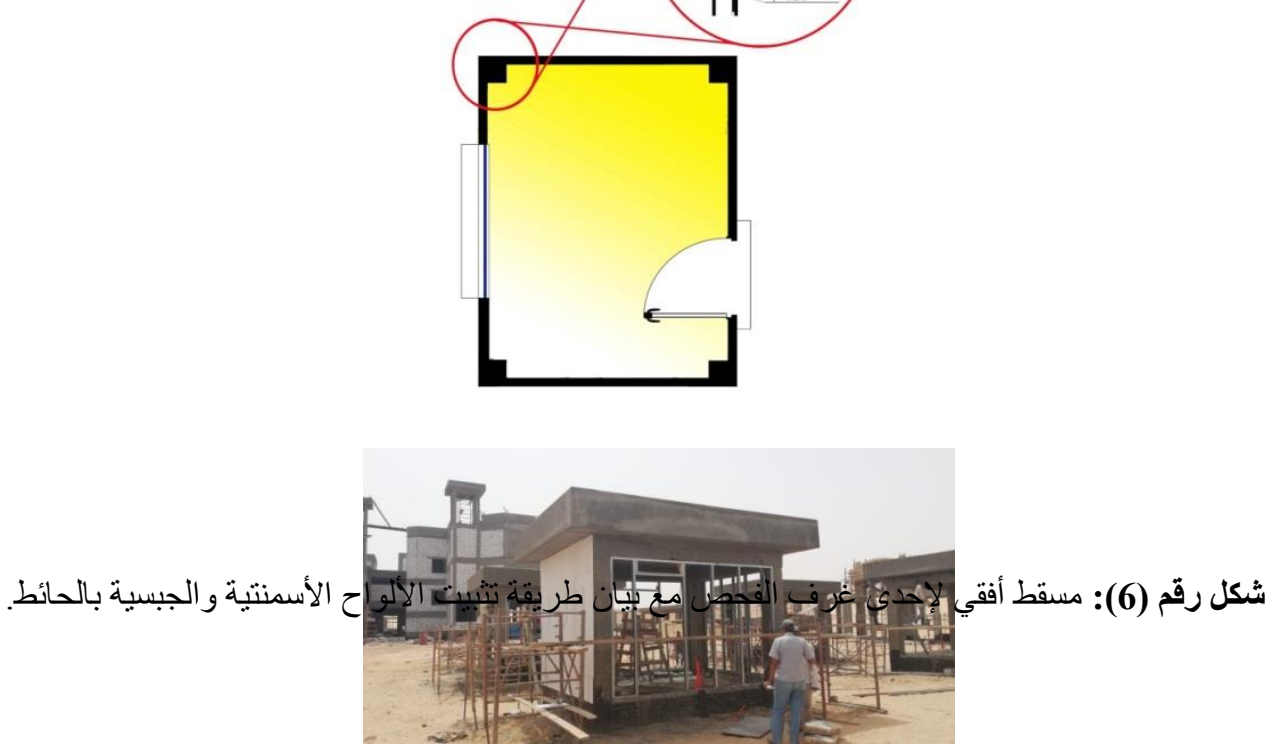

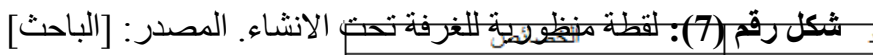

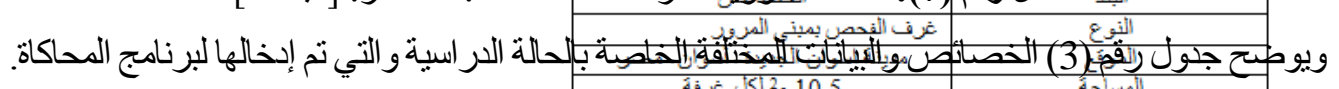

\begin{tabular}{|c|c|}
\hline 年 10.5 & 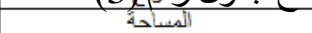 \\
\hline المستخدمة لنمل ذج الدر اسة. & جدول رئهن (3): البيانات \\
\hline 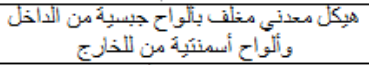 & الحوائط \\
\hline خرسانة مسلحة & الستف \\
\hline زجاج فردى 3مم & زجاج اللتبّاك \\
\hline 7 ساعاتَ من (8هباحَا حُّي 3 عصرا) & عدد ساعات الكمل \\
\hline فردين الكل غرفية & الآتشغال (فرد) \\
\hline نكيِف اسيلِبت & HVAC \\
\hline
\end{tabular}




\section{6. النتائج والمناقشة}

يقدم البحث ثلاث مقترحات لمعرفة مدي فعالية مادتي Bio PCM Q23 و Bio PCM Q25 عند دمجها بكل

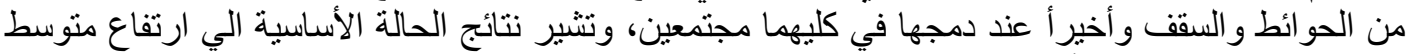

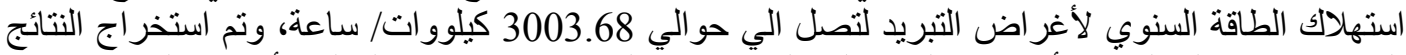

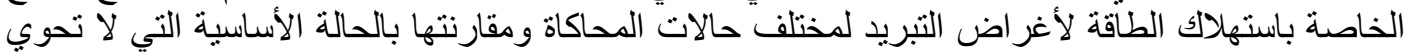

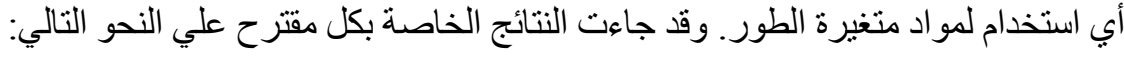

$$
\text { 1.6 المقتر ح الأول: دمج المواد متغبرة الطور بالحوائط الخارجية }
$$

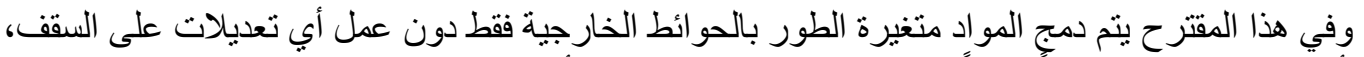

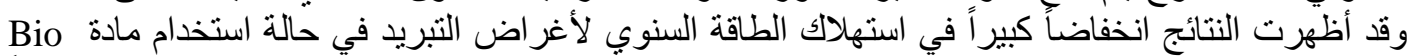

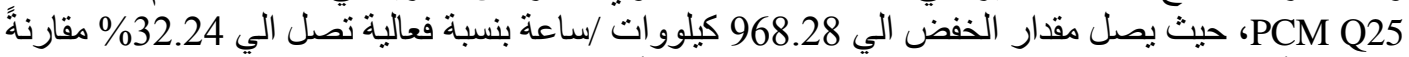

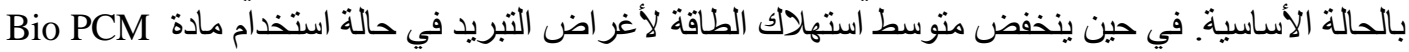
لتصل قيمة الخفض الي ف23.12 913 كيلووات /ساعة بفعالية تصل الي 30.4\%. ويوضح شكل رقم (8) النتائج الخاصة باستهلاك الطاقّة السنوي ومدي فاعلية هذه المو اد عند دمجهاً بالحو ائط.

$$
\text { 2.6. المقتر ح الثاني: دمج المواد متغبرة الطور بالسقف. }
$$

يتم دمج المو اد متغيرة الطور بالسقف فقط دون عمل أبي تعديلات على الحوائط الخارجية، وتشير النتائج الي انخفاض منوسط استهلاك الطاقة السنوي لأغراض التهبرة التبريد بقيمة

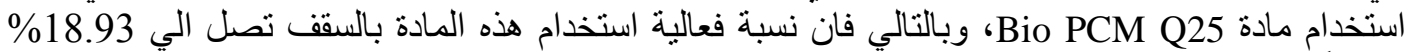

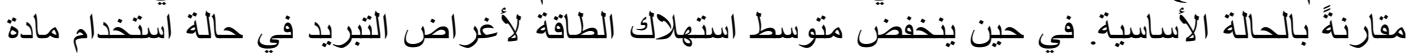
Bio PCM Q23

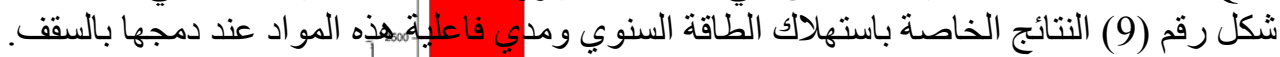
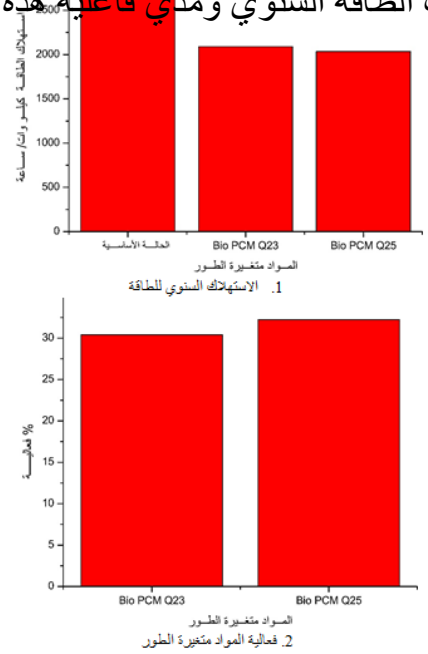

شكل رقم (8): دمج المو اد متغيرة الطور بالحو ائط 

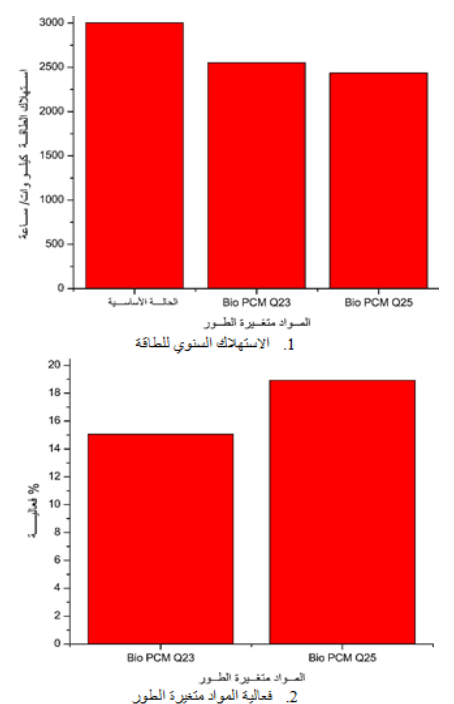

شكل رقم (9): دمج المو اد متغيرة الطور بالسقف

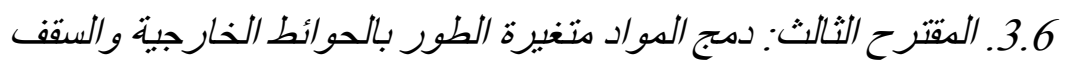

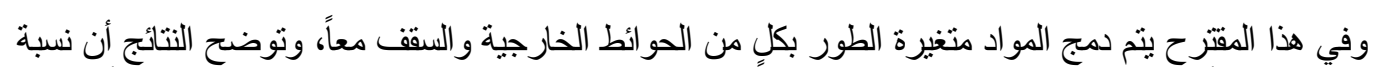

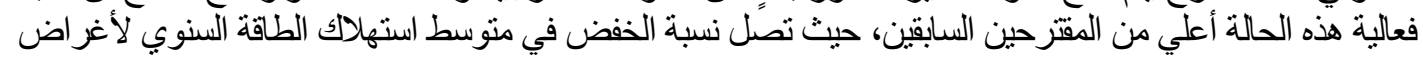

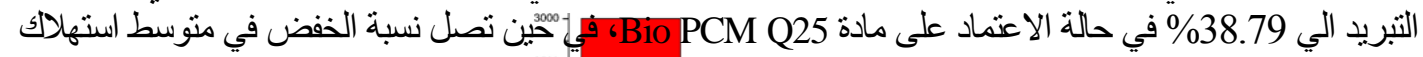

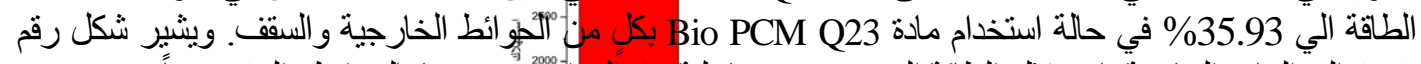

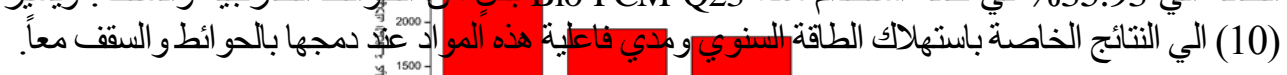

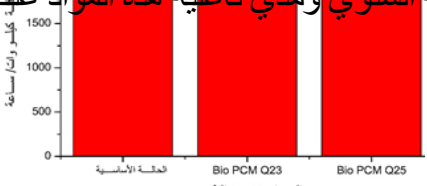

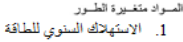

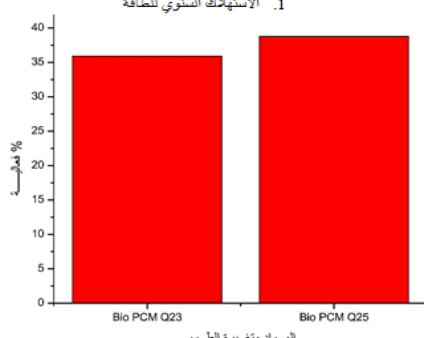

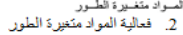

شكل رقم (10): دمج المو اد متغيرة الطور بالحو ائطو السقق. 
ينتهي البحث الي مدي قدرة مادتي Bio PCM Q23 و Bio PCM Q25 في خفض استهلاك الطاقة لأغر اض

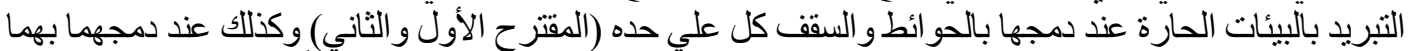

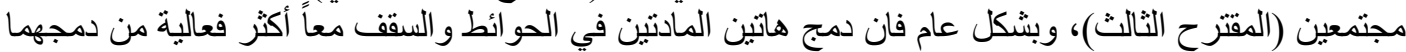

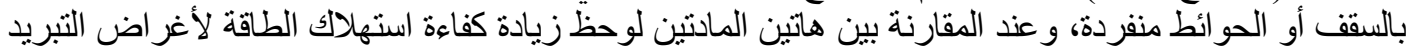

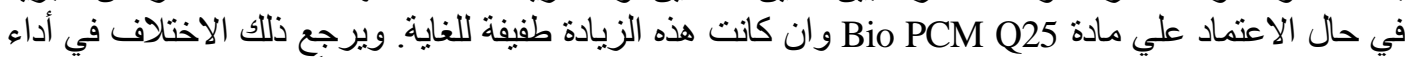

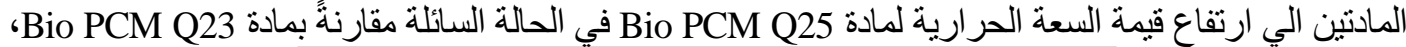

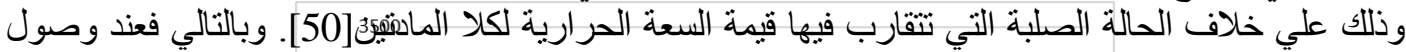

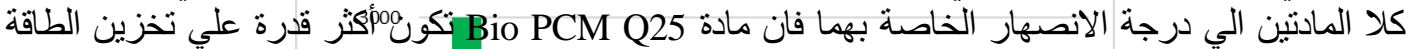

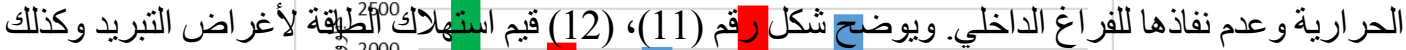
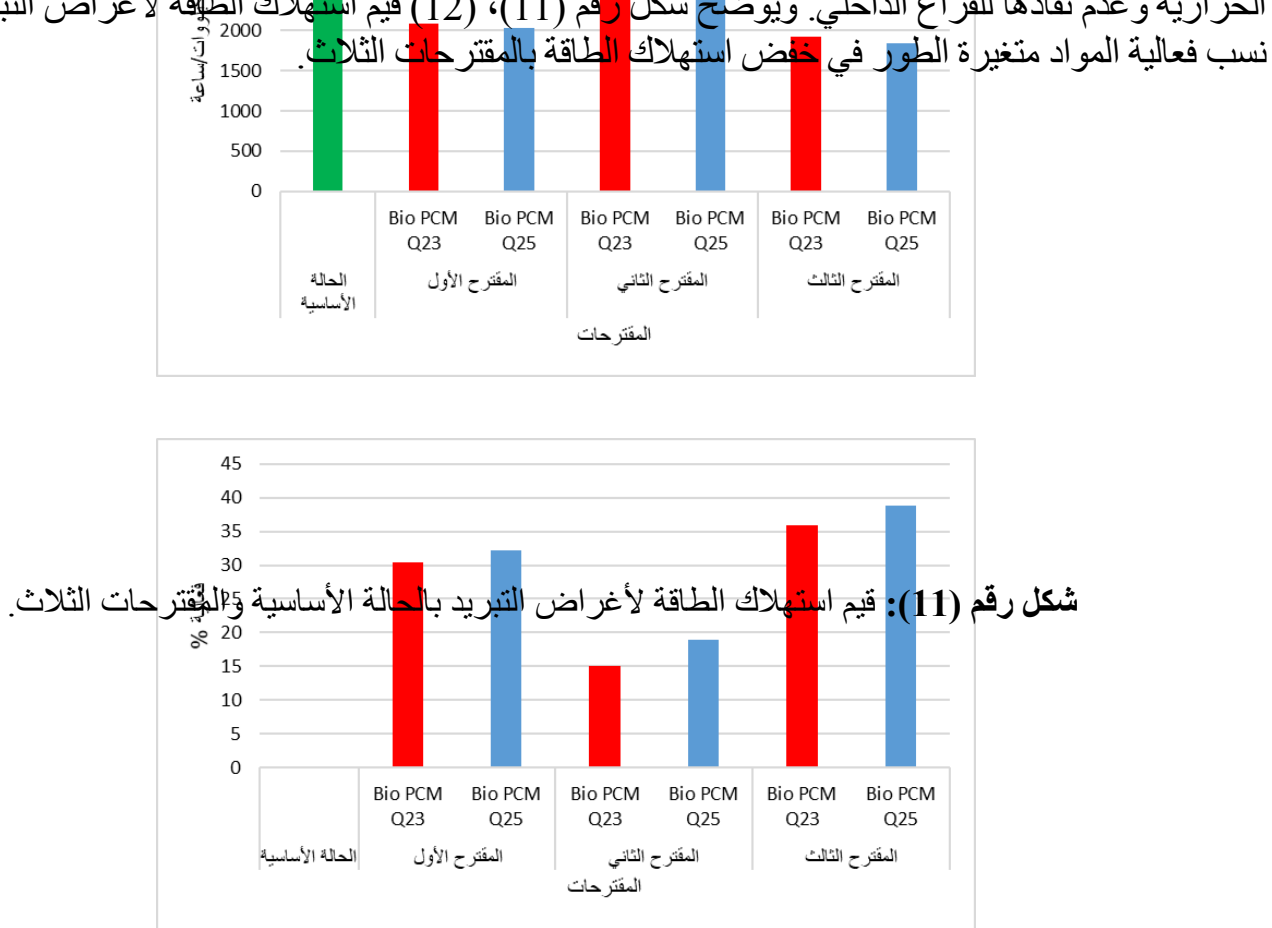

شكل رقم (12): نسبة فعالية المقترحات الثلاث في كفاءة استهلاك الطاقة لأغر اض التبريد.

و وند تحليل الأداء الحراري للغرفة في حال الاعتماد علي مادة Bio PCM Q25 ودون الاعتماد علي

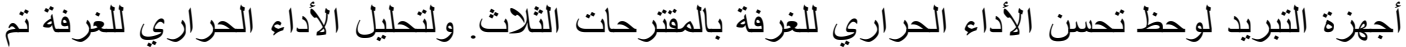

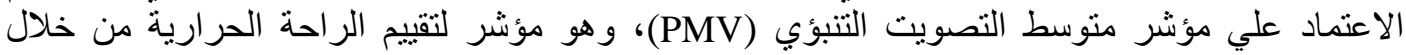
المعادلة التالية[51]

$\mathrm{PMV}=4+(0.303 \exp (-0.036 \mathrm{H})+0.0275) .\{6.57+0.46 \mathrm{H}+0.31 \mathrm{~Pa}+0.0017 \mathrm{HPa}$ $+0.0014 \mathrm{HTa}-4.13 \mathrm{fcl}(1+0.01 \mathrm{dT})(\mathrm{Tcl}-\mathrm{Tr})-\operatorname{hcfcl}(\mathrm{Tcl}-\mathrm{Ta})\}$ 
$\mathrm{Tcl}=35.7-0.0275 \mathrm{H}+0.155$ lclofcl $(4.13(1+0$. OldTemp $) 1+0.155$ lclofcl

$(4.13(1+0$. OldTemp) the

$\mathrm{Hc}=2.4(\mathrm{Tcl}-\mathrm{Ta}) 0.25$ or 12.1 square root of $\mathrm{v}, \mathrm{dt}=\mathrm{Tr}-22$

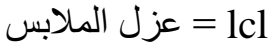

$$
\begin{aligned}
& \text { = fcr }
\end{aligned}
$$

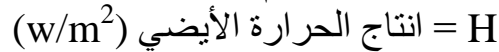

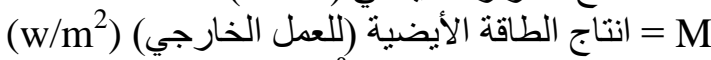

$$
\begin{aligned}
& \text { Ta } \\
& \text { (C) متو سط حر ارة الاشعاءع) Tr }
\end{aligned}
$$

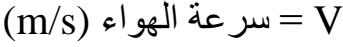

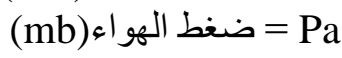

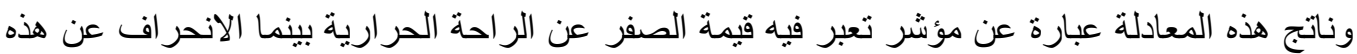

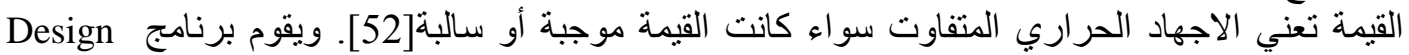

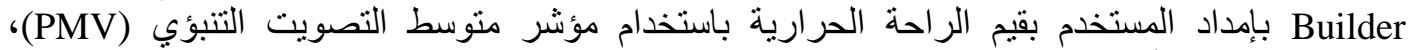

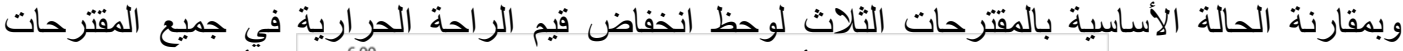

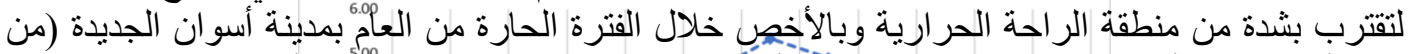

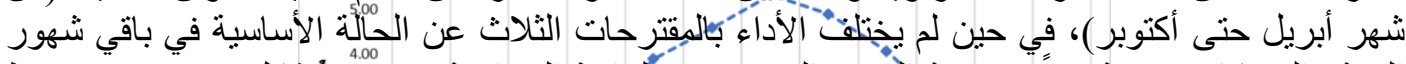

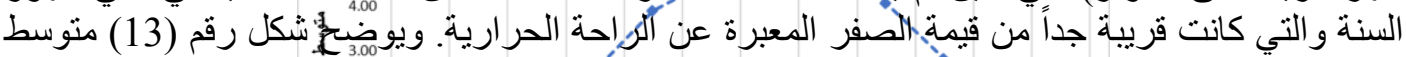

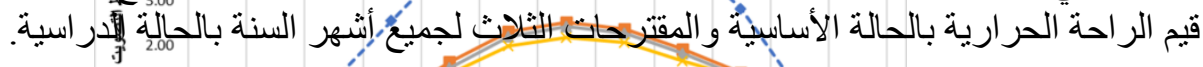

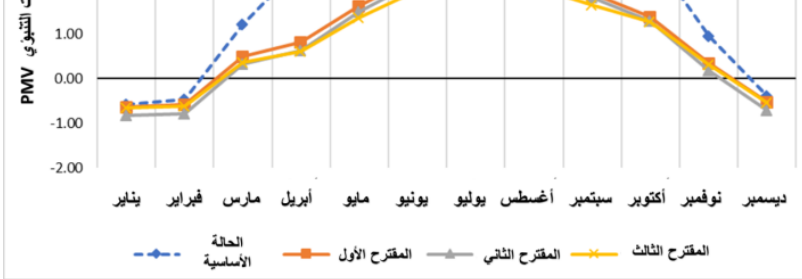

شكل رقم (13): متوسط قيم الراحة الحرارية بالحالة الأساسية والهترحات الثلاثث للحلة الدراسية.

8

بعد استعر اض النتائج السابقة يمكن القول بضرورة مر اعاة النقاط التالية:

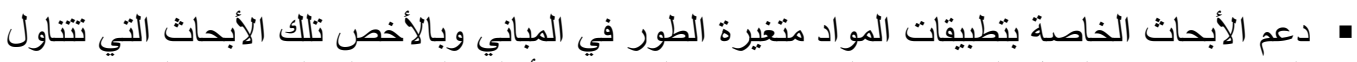

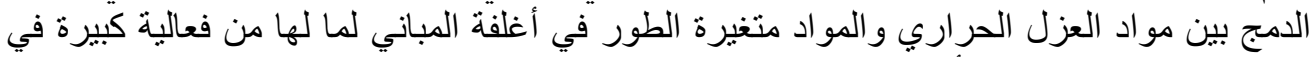

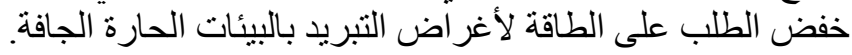

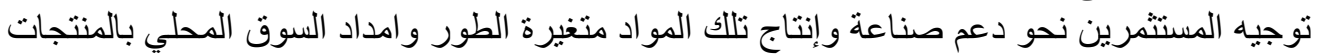

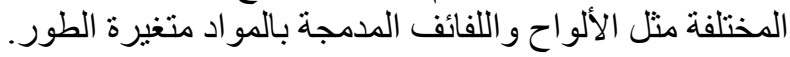

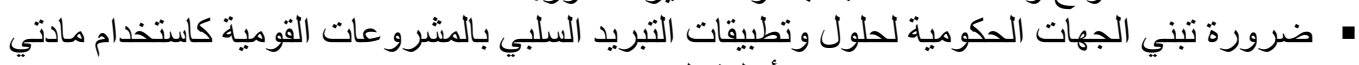
Bio PCM Q23 
• ضرورة المام طلبة وخريجي قسم العمارة بأهمية تلك المواد متغيرة الطور في خفض استهلاك الطاقة

لأغر اض التبريد وتحسين الظروف الظي المناخية بالفر اغات الداخلية.

المراجع

[1] M. Frigione, M. Lettieri, and A. Sarcinella, 'Phase Change Materials for Energy Efficiency in Buildings and Their Use in Mortars', Materials (Basel)., vol. 12, no. 8, p. 1260, Apr. 2019.

[2] S. B. Sadineni, S. Madala, and R. F. Boehm, 'Passive building energy savings: A review of building envelope components', Renewable and Sustainable Energy Reviews., vol. 15, no. 8, pp. 3617-3631, Oct. 2011.

[3] H. Akeiber et al., 'A review on phase change material (PCM) for sustainable passive cooling in building envelopes', Renewable and Sustainable Energy Reviews, vol. 60, pp. 1470-1497, Jul. 2016.

[4] Y. Konuklu, M. Ostry, H. O. Paksoy, and P. Charvat, 'Review on using microencapsulated phase change materials (PCM) in building applications', Energy and Buildings., vol. 106, pp. 134-155, Nov. 2015.

[5] 'DesignBuilder Software Ltd - Home'. [Online]. Available: https://designbuilder.co.uk/. [Accessed: 13-Aug-2019].

[6] 'Home - PCM - Phase Change Material'. [Online]. Available: https://www.pcmral.org/pcm/en/. [Accessed: 17-Jul-2019].

[7] A. de Gracia and L. F. Cabeza, 'Phase change materials and thermal energy storage for buildings', Energy and Buildings., vol. 103, pp. 414-419, Sep. 2015.

[8] B. T. Office, 'Energy Savings Potential and Research, Development, \&amp; Demonstration Opportunities for Residential Building Heating, Ventilation, and Air Conditioning Systems', Technical Report, Navigant Consulting, Inc., Burlington, MA (United States), 2012.

[9] K. O. Lee, M. A. Medina, E. Raith, and X. Sun, 'Assessing the integration of a thin phase change material (PCM) layer in a residential building wall for heat transfer reduction and management', Applied Energy, vol. 137, pp. 699-706, Jan. 2015.

[10] P. C. Tabares-Velasco, C. Christensen, and M. Bianchi, 'Verification and validation of EnergyPlus phase change material model for opaque wall assemblies', Building and Environment., vol. 54, pp. 186-196, Aug. 2012.

[11] X. Kong, C. Yao, P. Jie, Y. Liu, C. Qi, and X. Rong, 'Development and thermal performance of an expanded perlite-based phase change material wallboard for passive cooling in building', Energy and Buildings., vol. 152, pp. 547-557, Oct. 2017.

[12] A. Mavrigiannaki and E. Ampatzi, 'Latent heat storage in building elements: A systematic review on properties and contextual performance factors', Renewable and Sustainable Energy Reviews., vol. 60, pp. 852-866, Jul. 2016.

[13] D. Zhou, C. Y. Zhao, and Y. Tian, 'Review on thermal energy storage with phase change materials (PCMs) in building applications', Applied Energy, vol. 92, pp. 593-605, Apr. 2012.

[14] N. Soares, J. J. Costa, A. R. Gaspar, and P. Santos, 'Review of passive PCM latent heat thermal energy storage systems towards buildings' energy efficiency', Energy and Buildings., vol. 59, pp. 82-103, Apr. 2013.

[15] A. Waqas and Z. Ud Din, 'Phase change material (PCM) storage for free cooling of buildingsA review', Renewable and Sustainable Energy Reviews., vol. 18, pp. 607-625, Feb. 2013.

[16] P. Tatsidjodoung, N. Le Pierrès, and L. Luo, 'A review of potential materials for thermal energy storage in building applications', Renewable and Sustainable Energy Reviews., vol. 18, pp. 327-349, Feb. 2013.

[17] R. K. Sharma, P. Ganesan, V. V. Tyagi, H. S. C. Metselaar, and S. C. Sandaran, 'Developments in organic solid-liquid phase change materials and their applications in thermal energy storage', Energy Conversion and Management., vol. 95, pp. 193-228, May 2015.

[18] M. A. Wahid, S. E. Hosseini, H. M. Hussen, H. J. Akeiber, S. N. Saud, and A. T. Mohammad, 'An overview of phase change materials for construction architecture thermal management in hot and dry climate region', Applied Thermal Engineering., vol. 112, pp. 1240-1259, Feb. 2017.

[19] T. K. Aldoss, 'Maximizing the Usage of PCM Materials in Buildings', in ASME 2011 5th 


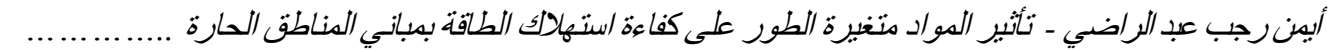

International Conference on Energy Sustainability, Parts A, B, and C, 2011, pp. 7-11.

[20] S. N. J. Al-Saadi, 'Phase Change Materials for Autonomous Energy Storage in Buildings Study of thermal energy storage using phase change materials View project Building Energy Audit for Improving Energy Efficiency and Integrating Solar System in a Library Building View project', 2015.

[21] S. N. Al-Saadi and Z. (John) Zhai, 'A new validated TRNSYS module for simulating latent heat storage walls', Energy and Buildings., vol. 109, pp. 274-290, Dec. 2015.

[22] R. Fikry, 'Application of phase change materials in residential buildings inner envelope', M.Sc thesis, Cairo University, 2016.

[23] S. Vazquez, S. M. Lukic, E. Galvan, L. G. Franquelo, and J. M. Carrasco, 'Energy Storage Systems for Transport and Grid Applications', IEEE Trans. Ind. Electron., vol. 57, no. 12, pp. 3881-3895, Dec. 2010.

[24] K. Pielichowska and K. Pielichowski, 'Phase change materials for thermal energy storage', Progress in Materials Science., vol. 65, pp. 67-123, Aug. 2014.

[25] Y. Sang, J. R. Zhao, J. Sun, B. Chen, and S. Liu, 'Experimental investigation and EnergyPlus-based model prediction of thermal behavior of building containing phase change material', Journal of Building Engineering., vol. 12, pp. 259-266, Jul. 2017.

[26] S. A. Memon, 'Phase change materials integrated in building walls: A state of the art review', Renewable and Sustainable Energy Reviews., vol. 31, pp. 870-906, Mar. 2014.

[27] I. Rahmanian, 'Thermal and Mechanical Properties of Gypsum Boards and Their Influences on Fire Resistance of Gypsum Board Based Systems', [Thesis]. Manchester, UK Univ. Manchester; 2011., Nov. 2011.

[28] Z. Wu, M. Qin, and M. Zhang, 'Phase change humidity control material and its impact on building energy consumption', Energy and Buildings., vol. 174, pp. 254-261, Sep. 2018.

[29] 'Phase Change Energy Solutions: Leader in Efficient Building Materials'. [Online]. Available: https://phasechange.com/. [Accessed: 15-Aug-2019].

[30] 'Phase Change Material Data Sheets I PCM Product Details'. [Online]. Available: https://phasechange.com/technology/data-sheets/. [Accessed: 16-Aug-2019].

[31] R. D. Beltrán and J. Martínez-Gómez, 'Analysis of phase change materials (PCM) for building wallboards based on the effect of environment', Journal of Building Engineering., vol. 24, p. 100726, Jul. 2019.

[32] A. Sarı, C. Alkan, and C. Bilgin, 'Micro/nano encapsulation of some paraffin eutectic mixtures with poly(methyl methacrylate) shell: Preparation, characterization and latent heat thermal energy storage properties', Applied Energy., vol. 136, pp. 217-227, Dec. 2014.

[33] L. F. Cabeza, A. Castell, C. Barreneche, A. de Gracia, and A. I. Fernández, 'Materials used as PCM in thermal energy storage in buildings: A review', Renewable and Sustainable Energy Reviews., vol. 15, no. 3, pp. 1675-1695, Apr. 2011.

[34] P. B. Salunkhe and J. K. D., 'Investigations on latent heat storage materials for solar water and space heating applications', Journal of Energy Storage, vol. 12, pp. 243-260, Aug. 2017.

[35] M. A. Nessim and S. A. Elariane, 'Review on Phase Change Materials and Its Applications in Buildings: Case Study of Egypt', SSRN Electron. J., Nov. 2017.

[36] J. Kosny, E. Kossecka, A. Brzezinski, A. Tleoubaev, and D. Yarbrough, 'Dynamic thermal performance analysis of fiber insulations containing bio-based phase change materials (PCMs)', Energy and Buildings., vol. 52, pp. 122-131, Sep. 2012.

[37] MennatAllah. Hassan. Youssef, 'The Impact of Integrating Phase Change Material upon Indoor Air Temperature in Hot Climates', M.Sc thesis, Faculty of Engineering, Cairo University, 2015.

[38 رامي جورج .محمد ياسين, كتأثير استخدام المو اد متغيرة الطور في الجنران و السقف في الأحمال الحرارية وورجة الحرارة

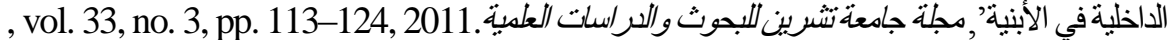

[39] مها أحمد. سهيل حنا, 'استخدام المواد متغيرة الطور لتخدئ لتخفيض احتياجات الطاقة في الأبنية السكنية', مجلة

$$
\text { جامعة البعث . vol. 39, no. 10, pp. 129-156, 2017 }
$$

[40] M. B. Al-Hadithi, 'Use of Phase Change Material in Residential Walls to Reduce Cooling Load', Anbar J. Eng. Sci. AJES-2011, vol. 4, no. 1.

[41] هاثم المحفوض, ،التحليل الحراري للجدران الحاوية علي المواد متغيرة الطور لاستخدامها في ترشيد 
JES, Assiut University, Faculty of Engineering, Vol. 48, No. 2, March 2020, pp. 302-316

استهلاك الطاقة في هياكل الأبنية', مجلة جامعة البعث. vol. 38, no. 8, pp. 113-141, 2016

[42] C. N.-M. David Beltrán, Isabel Miño-Rodríguez, Andrea Lobato, Andrés Gallardo, 'Thermal comfort performance within heritage buildings subject to a change of use', in Proceedings of the Mediterranean Green Building and Renewable Energy Forum 2015, 2015, pp. 1-6.

[43] R. Baetens, B. P. Jelle, and A. Gustavsen, 'Phase change materials for building applications: A state-of-the-art review', Energy and Buildings., vol. 42, no. 9, pp. 1361-1368, Sep. 2010.

[44] J. Lei, J. Yang, and E.-H. Yang, 'Energy performance of building envelopes integrated with phase change materials for cooling load reduction in tropical Singapore', Applied Energy, vol. 162, pp. 207-217, Jan. 2016.

[45] U. Berardi and M. Manca, 'The Energy Saving and Indoor Comfort Improvements with Latent Thermal Energy Storage in Building Retrofits in Canada', Energy Procedia, vol. 111, pp. 462-471, Mar. 2017.

[46] D. Beltran, J. Gómez Martínez, and A. Lobato Cordero, 'Effect of Environment on the Selection of Phase Change Materials for Building Wallboards Using Multi-criteria Decision Methods and Building Energy Simulations', 2017.

[47] 'Phase Change Material Data Sheets | PCM Product Details'. [Online]. Available: https://phasechange.com/technology/data-sheets/. [Accessed: 21-Jul-2019].

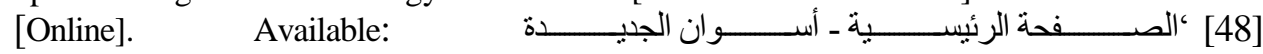

http://www.newcities.gov.eg/know_cities/Aswan/default.aspx. [Accessed: 17-Aug-2019].

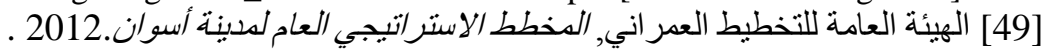

[50] D. Beltran, J. Martínez-Gómez, and A. Lobato-Cordero, 'Effect of environment on the selection of phase change materials for building wallboards using multi-criteria decision methods and Building Energy Simulations'.

[51] ASHRAE, 'Comfort. In: Handbook of Fundamentals.', Atlanta:8.1-8.29, 2001.

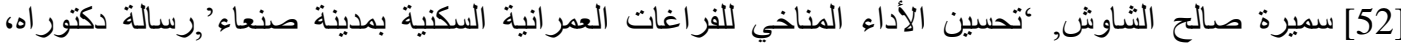

جامعة أسيوط, كلية الهندسة، قسم الهندسة المعمارية 2011.

\title{
THE IMPACT OF PHASE CHANGE MATERIALS ON THE BUILDINGS ENEGY EFFICIENCY IN THE HOT DESERT AREAS THE ANNEXED ROOMS OF THE TRAFFIC BUILDING IN NEW ASWAN CITY AS A CASE STUDY
}

\begin{abstract}
Energy crisis considered one of the most important challenges in the coming years due to future climate change and its negative effects as high values of air temperature and increasing energy demand for cooling purposes. In Egypt, the energy demand for cooling has been increased due to the increase in air temperature all over the republic. So, it became a very important issue to find a passive climatic solution. Phase Changing Materials (PCMs) considered as the best solution to decrease energy demand for cooling. Most international companies are currently competing in supporting the production of panels and rolls which depend on the integration with PCMs. This study depends on Design Builder software to evaluate the effect of Bio PCM Q23 and Bio PCM Q25 on energy consumption reduction. It was found that Bio PCM Q23 reduces the energy demand for cooling with an acceptable effectiveness percentage between $15.06 \%$ and $35.93 \%$ when combined with the building envelope, while the effectiveness of Bio PCM Q25 is between 18.93\% to $97.93 \%$ when combined with the same building envelope.
\end{abstract}

Keywords: Phase Change Materials, Energy efficiency, Building envelope, Design Builder. 